\title{
Co-morbid OSA and Depression- What we know and what we need to know
}

\section{Manvir Bhatia' and Saurav Khatiwada'}

1. Senior Consultant in Neurology and Sleep Medicine, Fortis Escorts Heart Institute and Research Centre Okhla New Delhi, Neurology and Sleep Centre in Hauz Khas Enclave New Delhi

2. MD Internal Medicine and Fellow in Sleep Medicine, Neurology and Sleep Centre in Hauz Khas Enclave New Delhi

DOI No: 10.5958/0974-0155.2016.00020.6

Indian J Sleep Med 2016; 11.4, 140-144

\begin{abstract}
Airway Centric Orthodontics is a philosophy which trumps everything else in contemporary Orthodontics. The philosophy focuses on practice of clinical orthodontics aimed at achieving ideal jaw relationship, establish normal oral function and performance, optimal proximal and occlusal contact of teeth. The central aspect of function and performance is airway and breathing which in fact is hierarchically the most important function for humans. Ideal health and ideal facial development is dependent on correct tongue posture and nasal breathing. Therefore contemporary protocols be it Preventive, interceptive or corrective orthodontics should factor upper airway improvement in addition to improving smile and facial appearance. Today Orthodontic profession is crucial and integral part of the interdisciplinary team in the management of upper airway sleep disorders, thus well poised to become a part of mainstream health profession. The paper would revisit the decision making process in orthodontics and discuss orthodontic strategies to improve the vital human airway which is essential for good health, longevity, and well-being.
\end{abstract}

Keywords: Airway, malocclusion, orthodontics

\section{Introduction}

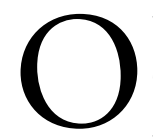
bstructive Sleep Apnea is a chronic sleep disorder with five or more apnea or hyponea per hour of sleep occurring due to collapse of upper airway despite the presence of central ventilatory drive ${ }^{1}$. It is usually a result of obesity or craniofacial

Address for correspondence:

\section{Dr. Manvir Bhatia}

Senior Consultant in Neurology and Sleep Medicine Fortis Escorts Heart Institute and Research Centre Okhla, New Delhi.

Neurology and Sleep Centre in Hauz Khas

Enclave, New Delhi

Email: neurologysleepcentre@gmail.com

Ph: 9654609951 malformations. Depression, on the other hand, is a psychiatric disorder of similar lifetime risk (8 to 12\% in most populations), multi-factorial origin with strong genetic and environmental predispositions ${ }^{2}$. OSA and depression have considerable overlap in both daytime and night-time symptomatology. Because there is a biological plausibility in the association of the two conditions which we shall delve in this article, chances of drawing spurious associations or prescribing inaccurate treatment are both possible. There have been studies looking into the relationship between the two conditions. In this article, we have summed up available literature to provide insight into the relationship between the two conditions so that clinical implications for sleep physicians, psychiatrists, psychologists and general practitioners can be drawn. 


\section{Epidemiology}

OSA has grown into a common health condition by the turn of the last century ${ }^{3-5}$. There has been an exponential growth in obesity all over the globe. As a result of multiple etiological factors, the prevalence of obstructive sleep apnea is also on a rise $e^{6}$. Few population-based studies place the prevalence of OSA at $4-7 \%$ for men and $2-4 \%$ for women ${ }^{3,4}$. An Indian study reports OSA prevalence to be as high as $7.5 \%$ in adult urban population ${ }^{4}$. The portion of OSA patients remaining untreated or undertreated is more than half. The age distribution of OSA shows gradual increase in incidence with age, peaking after 60 years of age ${ }^{3}$. Years may pass from the time of symptom onset before a diagnosis of OSA is made in most patients. These patients may suffer from depression over time due to increasing constrains they find in day to day living as well as due to metabolic derangements and other socio-economic factors.

\section{Depression In OSA}

An observational study in china ( $\mathrm{n}=1327$ OSA patients) showed prevalence of Depression as high as $47 \%$ on Symptom Checklist 90 and Self-Rating Depression Scale. The study revealed that depressive status is significantly associated with single status, high AHI i.e. OSA, and low family and social supports among those OSA patients.

In another study of newly diagnosed OSA patients $(\mathrm{n}=121)$ screened for Depression, prevalence of $44.6 \%$ on Beck Depression Inventory(BDI) score $(>10)$ was seen ${ }^{7}$. Another retrospective study of 100 OSA patients puts the proportion to $19 \%{ }^{8}$. This tendency is adequate a reason for sleep specialists to screen for Depression in an otherwise clear-cut OSA case. However, a causal relationship has not been established as further studies have shown that AHI values from sleep studies have not been shown to correlate with BDI scores (for depression $)^{7}$. So, only future studies can clarify whether or not a causal relationship exists in this significant association.

\section{OSA in Depression}

The prevalence of OSA in patients of major depressive disorder has been consistently reported to be high. In a recent systematic review for prevalence of OSA among psychiatry patients, data was pooled from 6 clinical studies $(n=522)$ with MDD'. A pooled prevalence of
OSA of $36.3 \%$ (95\% CI 19.4 to $57.4, \mathrm{p}<0.01$ ) was found. After excluding 2 studies (Carney 2006, Ong 2009 ) with potential selection bias, the pooled prevalence of OSA among 378 people with MDD was recalculated at $25.2 \%(95 \% \text { CI } 10.2 \text { to } 50.2, Q=58, \mathrm{p}<0.01)^{10,11}$. This was still higher than that reported for Schizophrenia and BPAD in the same review. In the review, an AHI/ $\mathrm{RDI}>5$ was taken as diagnostic for OSA while MDD was diagnosed based on DSM-IV (2000) or ICD-10 (1993), all being standard nomenclature. Increasing age and BMI were associated with OSA more significantly in these MDD patients on pooled analysis in the review.

\section{Possible Mechanisms for Association}

\section{Theory of Sleep fragmentation and hypoxia}

Beebe and Gozal (2002) came up with a theory that sleep fragmentation and hypoxia during sleep causes disruption of sleep-related restorative effects on the prefrontal cortex. This, in turn may result in 'executive dysfunction,' which refers to the abilities crucial for organization, planning, and adaptation ${ }^{12}$. In addition, a functional magnetic resonance imaging (fMRI) study has shown that OSA is associated with reduced lateral prefrontal activation during working memory tasks ${ }^{13}$. Similarly, functional neuroimaging abnormalities within the prefrontal cortex have also been associated with Major Depressive Disorders ${ }^{14,15}$.

The association of sleep fragmentation with depression has been evaluated in a study. This study reported significant increase in major flow limitations and desaturations in 19 depressed patients (compared to control subjects) selected without regard to OSA symptoms ${ }^{16}$. The authors hypothesized that respiratoryrelated sleep fragmentation of hypoxia-induced prefrontal dysfunction may predispose to mood disorder. But the lack of correlation of parameters of OSA and Depression i.e. AHI and BDI respectively as shown in at few studies argues against a causal relation ${ }^{7}$.

\section{Overlapping Symptomatology}

Another plausible hypothesis is that symptomatology of OSA and Depression overlap to cause the association. According to ICD-10, Depression is a symptom complex of persistent low mood and other somatic complaints, not attributed to the abuse of psychoactive substances 
or to an organic mental disorder, lasting for more than 2 weeks ${ }^{17}$. There is a considerable overlap between the somatic complaints of Depression and OSA. These depression symptoms are listed below in Table 1 comparing with similar symptoms that OSA patients can present with:

Considering the above scenario, patients with OSA syndrome may satisfy many somatic criteria for Depression and vise-versa, hence, an association.

\section{Socio-economic and Metabolic causes}

OSA patients may suffer from cognitive and memory decline along with constant daytime sleepiness and fatigue. Metabolic profile of untreated OSA patients show higher than normal prevalence of diabetes, dyslipidemia, hyperuricemia. There is also an increased incidence of hypertension and adverse cardiac events, heart failure and stroke in such patients. With these physical and mental disabilities, obesity with general inactivity, and their financial repercussions, OSA patients are under some psychological stress beyond normalcy. Biochemically, there is a constant rise in inflammatory cytokines like Il-6, TNF-alpha in both OSA and depression ${ }^{18,19}$. This may lead to increased incidence of depression over long term. On the other hand, depression is associated with incident and prevalent weight gain, especially in women ${ }^{20}$. Many patients also gain a significant amount of weight due to use of Tricyclic antidepressants, SSRIs or Anti-psychotics use. These patients can develop OSA while being on treatment. In this context, another hypothesis that - OSA and depression both have independent correlation with obesity (or metabolic syndrome) could explain their mutual correlation without dose-response relationship ${ }^{21}$.

\section{Therapeutic Trials on Management of Co-morbid OSA and Depression}

\section{Primarily OSA with Depressive Symptoms}

There have been some prospective cohort studies and fewer RCTs evaluating role of CPAP on depressive symptoms in OSA patients. A systematic review evaluated 26 of these open labelled studies which showed evidence in favour of improvement in depressive symptoms with adequate PAP therapy ${ }^{22}$. The evidence cannot be considered of high quality or of high effect size because of absence of double-blinded placebo controlled trials. In addition, a significant minority of trials still show no benefit of PAP in terms of depressive symptoms ${ }^{23-26}$.

In patients with residual sleepiness despite adequate management of OSA, it is a usual practice among sleep physicians to screen for depression if not done initially followed by behavioural and pharmacological treatment.

\section{Primarily Depression with OSA}

Though there is scarcity of studies addressing OSA in common cases of depression, some studies in resistant Depression have addressed OSA. In a study of 118 consecutive treatment resistant depression cases in Turkey, $27 \%$ were already diagnosed OSA and $11 \%$ more were found to be high risk for OSA ${ }^{27}$. These $38 \%$ patients benefitted significantly with treatment for OSA in terms of depressive symptoms. The authors concluded that in cases of Treatment Resistant Depression, especially with BMIe" $30 \mathrm{~kg} / \mathrm{m}^{2}$, presence of hypertension, and/or dyslipidemia, OSA should be positively screened for. However, placebo effect cannot be ruled out. Neverthe-less, treatment was effective and hazards of OSA were also managed which justifies screening and management for OSA in these subset of depressed patients.

\section{Summary of Evidences}

1. There is increased prevalence of OSA in Depressive disorder and vice-versa suggestive of a bidirectional association

2. There exists no dose-response relationship to signify a causal relationship

3. Treating for OSA may improve depressive symptoms significantly in most prospective cohort and pre-post studies but not in sham-controlled trials. This may imply either for a strong placebo effect in depression or hint towards depressive symptoms being a part of OSA itself, primary depression not being associated altogether.

OSA and Depression have a complex etiological and demographical overlap with similar symptomatology. At this point, we know that both the conditions have an association that requires enquiring for the other in presence of one. We do not yet know the exact reason for the significant association. Most likely, it is a multifactorial association with each of the above hypothesis being applicable in various subsets of patients. It is 
therefore necessary to appreciate the bidirectional relationship and be vigilant at both ends in order to quickly diagnose and treat the co-morbidities.

\section{Conclusion}

\section{Research Perspective}

Studies showing clear treatment benefit in OSA complicated by depression are lacking. Hence, doubleblinded studies comparing effects of PAP therapy with placebo versus PAP therapy with anti-depressants in patients of OSA with Comorbid Depression may provide insight into the therapeutic implications of this association. The outcomes to look for in these studies should be time to and extent of restoration of EDS, fatigue and other symptoms, safety outcomes like weight gain, and compliance to treatment. The study should stratify cases of depression based on whether they are ${ }^{1}$ primary depression ${ }^{2}$, reactive to OSA-induced psychosocial stressors ${ }^{3}$, Severe OSA induced excessive sleepiness and cognitive impairment or $^{4}$ other stressor induced causes. Only with all this information, we can have a clearer idea into depression complicating OSA.

More studies to look for OSA (with appropriate screening questionnaires followed by Sleep study) in new and treatment resistant patients of depression should be carried out to identify significant predictors of OSA in depressed patients.

Studies should also focus on protocol to identify and manage depression induced residual sleepiness in OSA patients on PAP therapy. Likewise, sham controlled RCTs evaluating PAP therapy on resistant depression cases with mild OSA or flow-limitation will help identify the importance of screening for sleep-disordered breathing in the patient group.

\section{Clinical Management Perspective}

An OSA patient with co-morbid depression is unlikely to show resilience to withstand fear of PAP therapy. $\mathrm{He} /$ she may soon become non-compliant to PAP therapy. Therefore, a vigilant search for depressive symptoms, arrangement of psychological evaluation followed by watchful waiting in mild cases to counselling / drug therapy based on psychiatry opinion is prudent.

From psychiatry practice point of view, a patient identified with depression only and untreated for OSA may not gain any respite from his fatigue and lack of interest, but rather suffer from side effects of TCAs or SSRIs. Likewise, a previously responding patient of Depression may become resistant to drug treatment after significant weight gain and development of OSA. Therefore, patients with depression and excessive daytime sleepiness or fatigue must be screened with OSA screening questionnaires. Appropriate referrals for sleep study should be made based on the same. Periodic reassessment for OSA is required because of the dynamics of obesity in these cases.

\section{References}

1. Berry RB, Budhiraja R, Gottlieb DJ, Gozal D, Iber C, Kapur VK, et al. Rules for Scoring Respiratory Events in Sleep: Update of the 2007 AASM Manual for the Scoring of Sleep and Associated Events. J Clin Sleep Med JCSM Off Publ Am Acad Sleep Med. 2012 Oct 15;8(5):597-619.

2. Andrade L, Caraveo-Anduaga JJ, Berglund P, Bijl RV, De Graaf R, Vollebergh W, et al. The epidemiology of major depressive episodes: results from the International Consortium of Psychiatric Epidemiology (ICPE) Surveys. Int J Methods Psychiatr Res. 2003;12(1):3-21.

3. Young T, Palta M, Dempsey J, Skatrud J, Weber S, Badr S. The occurrence of sleep-disordered breathing among middle-aged adults. N Engl J Med. 1993 Apr $29 ; 328(17): 1230-5$.

4. Udwadia ZF, Doshi AV, Lonkar SG, Singh CI. Prevalence of Sleep-disordered Breathing and Sleep Apnea in Middleaged Urban Indian Men. Am J Respir Crit Care Med. 2004 Jan 15;169(2):168-73.

5. Bixler EO, Vgontzas AN, Ten Have T, Tyson K, Kales A. Effects of age on sleep apnea in men: I. Prevalence and severity. Am J Respir Crit Care Med. 1998 Jan;157(1):144-8.

6. Peppard PE, Young T, Barnet JH, Palta M, Hagen EW, Hla KM. Increased Prevalence of Sleep-Disordered Breathing in Adults. Am J Epidemiol. 2013 May 1;177(9):1006-14.

7. McCall WV, Harding D, O'Donovan C. Correlates of depressive symptoms in patients with obstructive sleep apnea. J Clin Sleep Med JCSM Off Publ Am Acad Sleep Med. 2006 Oct 15;2(4):424-6.

8. Pinto JA, Ribeiro DK, Cavallini AF da S, Duarte C, Freitas GS. Comorbidities Associated with Obstructive Sleep Apnea: a Retrospective Study. Int Arch Otorhinolaryngol. 2016 Apr;20(2):145-50.

9. Stubbs B, Vancampfort D, Veronese N, Solmi M, Gaughran $\mathrm{F}$, Manu P, et al. The prevalence and predictors of obstructive sleep apnea in major depressive disorder, bipolar disorder and schizophrenia: A systematic review and meta-analysis. J Affect Disord. 2016 Jun;197:259-67.

10. Carney RM, Howells WB, Freedland KE, Duntley SP, Stein PK, Rich MW, et al. Depression and obstructive sleep apnea in patients with coronary heart disease. Psychosom Med. $2006 ; 68(3): 443-8$. 
11. Ong JC, Gress JL, San Pedro-Salcedo MG, Manber R. Frequency and predictors of obstructive sleep apnea among individuals with major depressive disorder and insomnia. J Psychosom Res. 2009;67(2):135-41.

12. Beebe DW, Gozal D. Obstructive sleep apnea and the prefrontal cortex: towards a comprehensive model linking nocturnal upper airway obstruction to daytime cognitive and behavioral deficits. J Sleep Res. 2002 Mar;11(1):1-16.

13. Thomas RJ, Rosen BR, Stern CE, Weiss JW, Kwong KK. Functional imaging of working memory in obstructive sleepdisordered breathing. J Appl Physiol Bethesda Md 1985. 2005 Jun;98(6):2226-34.

14. Drevets WC. Functional anatomical abnormalities in limbic and prefrontal cortical structures in major depression. Prog Brain Res. 2000;126:413-31.

15. Videbech P. PET measurements of brain glucose metabolism and blood flow in major depressive disorder: a critical review. Acta Psychiatr Scand. 2000 Jan;101(1): 11-20.

16. Deldin PJ, Phillips LK, Thomas RJ. A preliminary study of sleep-disordered breathing in major depressive disorder. Sleep Med. 2006 Mar;7(2):131-9.

17. World Health Organization. The ICD-10 classification of mental and behavioural disorders: clinical descriptions and diagnostic guidelines. 1st ed. Geneva: World Health Organization.; 1992.

18. Miller GE, Stetler CA, Carney RM, Freedland KE, Banks WA. Clinical depression and inflammatory risk markers for coronary heart disease. Am J Cardiol. 2002 Dec $15 ; 90(12): 1279-83$.

19. Lanquillon S, Krieg JC, Bening-Abu-Shach U, Vedder H. Cytokine production and treatment response in major depressive disorder. Neuropsychopharmacol Off Publ Am Coll Neuropsychopharmacol. 2000 Apr;22(4):370-9.
20. Singh G, Jackson CA, Dobson A, Mishra GD. Bidirectional association between weight change and depression in midaged women: a population-based longitudinal study. Int J Obes. 2014 Apr 1;38(4):591-6.

21. De Wit L, Luppino F, van Straten A, Penninx B, Zitman F, Cuijpers P. Depression and obesity: a meta-analysis of community-based studies. Psychiatry Res. $2010 \mathrm{Jul}$ $30 ; 178(2): 230-5$.

22. McMahon JP, Foresman $B H, C h i s h o l m ~ R C$. The influence of CPAP on the neurobehavioral performance of patients with obstructive sleep apnea hypopnea syndrome: a systematic review. WMJ Off Publ State Med Soc Wis. $2003 ; 102(1): 36-43$.

23. Yu BH, Ancoli-Israel S, Dimsdale JE. Effect of CPAP treatment on mood states in patients with sleep apnea. J Psychiatr Res. 1999 Oct;33(5):427-32.

24. Henke KG, Grady JJ, Kuna ST. Effect of nasal continuous positive airway pressure on neuropsychological function in sleep apnea-hypopnea syndrome. A randomized, placebocontrolled trial. Am J Respir Crit Care Med. 2001 Mar;163(4):911-7.

25. Borak J, Cieœlicki JK, Koziej M, Matuszewski A, Zieliñski J. Effects of CPAP treatment on psychological status in patients with severe obstructive sleep apnoea. J Sleep Res. 1996 Jun;5(2): 123-7.

26. Muñoz A, Mayoralas LR, Barbé F, Pericás J, Agusti AG. Long-term effects of CPAP on daytime functioning in patients with sleep apnoea syndrome. Eur Respir J. 2000 Apr;15(4):676-81.

27. Fitgerald CT, Hiett E, Buysee DJ, Altýntap N, Aikman G, Fýrat H. Sleep Apnea in a Sample of Patients with Treatment Resistant Depression Referred for Electroconvulsive Therapy. J Turk Sleep Med. 2015 Mar 5;2(1):7-10. 